\title{
REGENERAÇÃO NATURAL DO COMPONENTE ARBÓREO APÓS A MORTALIDADE DE UM MACIÇO DE TAQUARA EM UM FRAGMENTO DE FLORESTA OMBRÓFILA MISTA EM LAGES - SC
}

\author{
TREE SPECIES NATURAL REGENERATION AFTER A MASSIVE BAMBOO DIE-OFF IN AN \\ ARAUCARIA FOREST FRAGMENT IN LAGES, SANTA CATARINA STATE
}

\begin{abstract}
Kristiana Fiorentin dos Santos ${ }^{1}$ Tiago de Souza Ferreira ${ }^{2}$ Pedro Higuchi ${ }^{3}$ Ana Carolina da Silva ${ }^{4}$ Paula Búrigo Vandresen ${ }^{5}$ Adriano da Costa $^{1}$ Grasiela Spada $^{6}$ Verônica Schmitz ${ }^{7}$ Franciele de Souza
\end{abstract}

\section{RESUMO}

O presente trabalho teve como objetivo analisar a composição florística e a estrutura da regeneração natural em um setor onde ocorreu a mortalidade sincronizada de um maciço de taquara (Merostachys multiramea Hackel) (CT) e em uma área adjacente, com dossel contínuo e ausência de taquara (ST), em um fragmento de Floresta Ombrófila Mista Montana em Lages - SC. Foram alocadas 14 unidades amostrais de 5 x $5 \mathrm{~m}$ (seis no setor CT e oito no setor $\mathrm{ST}$ ), nas quais todos os indivíduos arbóreos regenerantes com circunferência a altura do peito (CAP) $\leq 15 \mathrm{~cm}$ e altura $\geq 25 \mathrm{~cm}$ foram identificados e mensurados (diâmetro do colo). As famílias com maior riqueza de espécies foram: Myrtaceae (nove), Solanaceae (seis) e Aquifoliaceae (quatro). O setor ST apresentou um índice de diversidade de Shannon de 2,73 e o setor CT apresentou o valor de 2,31. Solanum variabile, Piptocarpha angustifolia, Mimosa scabrella, Jacaranda puberula e Solanum pseudoquina foram as espécies de maior valor de importância (VI) no setor CT. As espécies de maior VI no setor ST foram Myrsine lorentziana, Casearia decandra, Cinnamodendron dinisii, Drimys brasiliensis e Ilex paraguariensis. Os resultados mostraram que a mortalidade sincronizada de indivíduos de taquara influenciou na variação espacial dos descritores florísticos e estruturais da regeneração natural de espécies arbóreas.

Palavras-chave: Merostachys multiramea; componente regenerante; descritores fitossociológicos.

\section{ABSTRACT}

The present study aimed to analyze the floristic and structural descriptors of tree species natural regeneration

1 Engenheiro Florestal, Mestrando em Ciência do Solo, Centro de Ciências Agroveterinárias, Universidade do Estado de Santa Catarina, Av. Luiz de Camões, 2090, Conta Dinheiro, CEP 88520-000, Lages (SC), Brasil. kristianafiorentin@yahoo.com.br/adrianodacosta23@yahoo.com.br

2 Engenheiro Florestal, Mestrando em Engenharia Florestal, Centro de Ciências Agroveterinárias, Universidade do Estado de Santa Catarina, Av. Luiz de Camões, 2090, Conta Dinheiro, CEP 88520-000, Lages (SC), Brasil. tiagoferreira@florestal.eng.br

3 Engenheiro Florestal, Dr., Professor do Departamento de Engenharia Florestal, Centro de Ciências Agroveterinárias, Universidade do Estado de Santa Catarina, Av. Luiz de Camões, 2090, Conta Dinheiro, CEP 88520-000, Lages (SC), Brasil. higuchip@gmail.com

4 Engenheira Florestal, Dr ${ }^{\mathrm{a}}$., Professora do Departamento de Engenharia Florestal, Centro de Ciências Agroveterinárias, Universidade do Estado de Santa Catarina, Av. Luiz de Camões, 2090, Conta Dinheiro, CEP 88520-000, Lages (SC), Brasil. carol_sil4@yahoo.com.br

5 Engenheira Florestal, Mestranda em Ciências Florestais, Universidade Estadual do Centro-Oeste, BR 153, Km 7, Riozinho CEP 84500-000, Irati (PR), Brasil. paulabph@hotmail.com

6 Engenheira Florestal, Mestranda em Ciência Florestal, Faculdade de Ciências Agronômicas, Seção de PósGraduação Fazenda Experimental Lageado, Universidade Estadual Paulista, Rua José Barbosa de Barros, 1780, CEP 18610-307, Botucatu (SP), Brasil. gra_spada@hotmail.com

7 Engenheira Florestal, Centro de Ciências Agroveterinárias, Universidade do Estado de Santa Catarina, Av. Luiz de Camões, 2090, Conta Dinheiro, CEP 88520-000, Lages (SC), Brasil. vschmitz883@hotmail.com/ fran28souza@hotmail.com

Recebido para publicação em 20/04/2011 e aceito em 4/06/2013

Ci. Fl., v. 25, n. 1, jan.-mar., 2015 
in a forest sector with synchronized bamboo (Merostachys multiramea Hackel ) die-off (CT) and an adjacent area with continuous canopy cover (ST) in an araucaria forest fragment in the municipality of Lages, Santa Catarina state. A total of $14,5 \times 5 \mathrm{~m}$, plots (six plots in CT sector and eight in ST sector) were allocated, where all tree species regenerative individual with circumference at breast height smaller than $15 \mathrm{~cm}$ and height higher than $25 \mathrm{~cm}$ was measured (diameter at soil level) and identified. The richest families were: Myrtaceae (nine), Solanaceae (six) and Aquifoliaceae (four). The Shannon Diversity Index in ST and CT sectors were respectively 2.73 and 2.31. The species with the highest importance values in CT sector were Solanum variabile, Piptocarpha angustifolia, Mimosa scabrella, Jacaranda puberula and Solanum pseudoquina. In ST sector, the species with highest importance values were Myrsine lorentziana, Casearia decandra, Cinnamodendron dinisii, Drimys brasiliensis and Ilex paraguariensis. The results showed that the synchronized bamboo die-off influenced the spatial variation in the floristic and structural descriptors of the tree species natural regeneration.

Keywords: Merostachys multiramea; regenerative component; phytosociological descriptors.

\section{INTRODUÇÃO}

Estudos sobre a regeneração natural em florestas são de grande relevância para o entendimento do funcionamento ecológico desses ecossistemas, pois permitem a inferência sobre a dinâmica de comunidades e populações de espécies arbóreas, que representa uma informação fundamental para o manejo florestal. No entanto, entender o padrão da regeneração de espécies arbóreas é uma tarefa complexa, pois esta depende da interação entre fatores intrínsecos e extrínsecos, relacionada às características ecológicas das espécies e às condições ambientais. Além disso, muitos eventos determinantes, como a formação de clareiras e a dispersão de sementes, ocorrem de forma estocástica, o que aumenta a complexidade de todo o processo em ambientes naturais.

Vários fatores podem explicar as variações espaciais e temporais de parâmetros florísticos e estruturais do componente regenerativo no sub-bosque de florestas como, por exemplo, a formação de clareiras naturais (BROKAW, 1987), a intensidade de luz (MONTGOMERY e CHAZDON, 2002), a distância da fonte de propágulo (HIGUCHI et al., 2010), a presença de lianas (SCHNITZER et al., 2000) e a fertilidade do solo (VEENENDAAL et al., 1996). Além desses fatores, estudos em florestas tropicais e subtropicais no Brasil (GUILHERME et al., 2004; BUDKE et al., 2010) e em diversas partes do mundo (MAROD et al., 1999; LARPKERN et al., 2011) também têm demonstrado que a presença de maciços de bambu ou taquara no interior florestal desempenha importante papel na regeneração de espécies arbóreas, afetando padrões de diversidade, composição florística, estrutura e a dinâmica do componente regenerativo.

Estes maciços, popularmente conhecidos como bambuzal ou taquaral, são formados por espécies da família Poaceae e ocorrem na forma de manchas no interior de florestas, principalmente em áreas perturbadas (SCHMIDT, 2008). No interior dessas manchas, a regeneração natural é inibida (MAROD et al., 1999) devido à competição interespecífica, acúmulo de serrapilheira sobre o solo e redução da luminosidade (LARPKERN et al., 2011). Uma característica importante deste grupo de plantas se refere ao fato de apresentar grande intervalo entre os eventos reprodutivos, como, por exemplo, 30 a 34 anos para Merostachys skvortzovii (LIEBSCH e REGINATO, 2009). Diante disso, em fragmentos florestais degradados, o controle da taquara pode ser considerado como uma estratégia de restauração ecológica. No entanto, o florescimento e a posterior morte sincronizada destes elementos provoca uma grande mudança ambiental (BUDKE et al., 2010; LARPKERN et al., 2011), abrindo uma oportunidade de regeneração para espécies vegetais que apresentam outros hábitos de vida, como as árvores.

Considerando que o florescimento e a posterior mortalidade do taquaral são acontecimentos difíceis de serem observados, devido ao longo tempo entre os eventos reprodutivos, estes eventos representam oportunidades únicas para investigações da ecologia florestal. Desta forma, com o propósito de conhecer a regeneração natural após esse evento pouco frequente e compreender melhor o funcionamento da Floresta Ombrófila Mista, o objetivo deste trabalho foi analisar a 
composição florística e a estrutura do componente regenerativo de espécies arbóreas após a mortalidade sincronizada do maciço de taquara (Merostachys multiramea Hackel) e comparar com a regeneração natural que ocorre em área adjacente sem taquara, sob o dossel contínuo do componente adulto em um fragmento florestal em Lages - SC.

\section{MATERIAL E MÉTODO}

$\mathrm{O}$ estudo foi realizado em um fragmento florestal de 234,42 ha e altitude de 884 m localizado no município de Lages - SC, no Parque Natural Municipal João José Theodoro da Costa Neto PARNAMUL (2781'S e $\left.50^{\circ} 32^{\prime} \mathrm{W}\right)$. A vegetação do fragmento, segundo a classificação do IBGE (1992), é Floresta Ombrófila Mista Montana. A precipitação média anual na região é de 1200-1900 $\mathrm{mm}$, com chuvas bem distribuídas durante o ano, e a temperatura média anual é de $15-16^{\circ} \mathrm{C}$. O clima, segundo a classificação de Köppen, é Cfb.

Foram estudados dois setores na floresta: um onde ocorreu a mortalidade do maciço de taquara (Merostachys multiramea Hackel) (CT), entre os anos de 2005 e 2006, e outro sob o dossel contínuo do componente arbóreo (ST), sem resquícios de taquara. No ano de 2010, no setor CT foram alocadas seis parcelas e no setor ST foram alocadas oito parcelas, totalizando 14 unidades amostrais (Figura 1). As parcelas quadrangulares foram alocadas de forma contínuas e cada uma tinha 25 $\mathrm{m}^{2}$.

Dentro de cada parcela, no mesmo ano, todos os regenerantes de espécies arbóreas com CAP (circunferência a altura do peito) $\leq 15 \mathrm{~cm}$ e altura $\geq 25 \mathrm{~cm}$ foram inventariados. Foi mensurado o diâmetro do colo, com auxílio de um paquímetro, e determinada a identificação botânica dos indivíduos amostrados. As espécies foram classificadas em grupos ecológicos, de acordo com a classificação proposta por Swaine e Whitmore (1988), como sendo pioneiras, clímax exigentes de luz e clímax tolerantes à sombra.

Para verificar se o perfil florístico foi adequadamente representado, foi elaborada a curva de acumulação de espécies. Essa curva foi construída por meio do método de aleatorização, com 1000 permutações. A distribuição dos valores de riqueza estimados para as unidades amostrais, por meio das permutações, foi inserida na curva de acumulação de espécies, utilizando gráficos do tipo "boxplot".

A diversidade de cada setor inventariado foi quantificada por meio do Índice de Shannon (H'). Para conhecimento da dominância ecológica também foi calculada a Equabilidade de Pielou (J). A riqueza entre setores foi comparada por meio da técnica de rarefação, considerando a mesma quantidade de indivíduos para ambos os setores. Neste caso, o número de indivíduos utilizado na rarefação foi definido como sendo aquele encontrado no setor de menor densidade (209 indivíduos). A estrutura do componente arbóreo foi descrita a partir do cálculo dos descritores quantitativos clássicos propostos por Mueller-Dombois e Ellemberg (1974): densidade absoluta e relativa, frequência

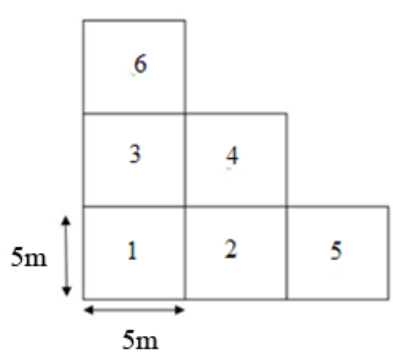

CT

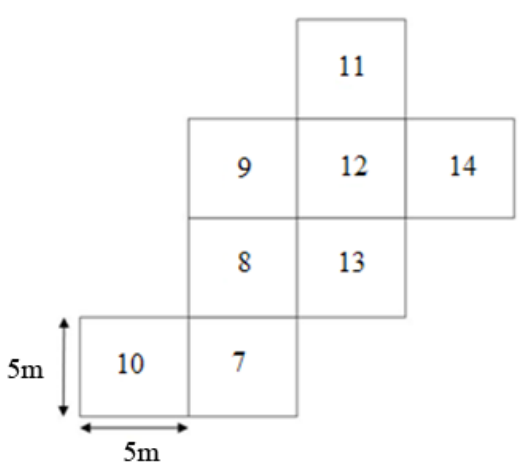

ST

FIGURA 1: Distribuição das parcelas nos setores da floresta onde ocorreu a mortalidade de taquara (CT) e com o dossel contínuo do componente arbóreo (ST) em um fragmento de Floresta Ombrófila Mista, em Lages - SC.

FIGURE 1: Plots distribution in sectors with bamboo die-off (CT) and with tree component continuous canopy (ST) in an Araucaria Forest fragment, in the municipality of Lages, SC state. 
absoluta e relativa, dominância absoluta e relativa e valor de importância (VI).

Para verificar a existência de variações estruturais e florísticas, entre os dois setores, foi realizada uma Análise de Correspondência Retificada (DCA, "Detrendend Correspondence Analyses"), utilizando uma matriz de abundância das espécies nas parcelas de ambos os setores. Todas as análises foram realizadas utilizando a planilha eletrônica Microsoft Office Excel e o programa $R$ (R DEVELOPMENT CORE TEAM, 2010), com suporte das bibliotecas Vegan (OKSANEN et al., 2010), para a análise multivariada e a curva de acumulação de espécies, e Rich (ROSSI, 2011), para a rarefação.

\section{RESULTADOS E DISCUSSÃO}

Nas parcelas inventariadas foram encontrados 527 indivíduos regenerantes de espécies arbóreas, pertencentes a 51 espécies, 35 gêneros e 25 famílias botânicas (Tabela 1). Deste total, uma espécie foi identificada somente em nível de família e duas em nível de gênero. Além das espécies encontradas, seis indivíduos presentes na área não puderam ser identificados por não apresentarem folhas.

O número de espécies encontrado no presente estudo (51) foi similar ao encontrado por outros autores avaliando a regeneração natural em fragmentos de Floresta Ombrófila Mista, como Caldato et al. (1996) em Caçador - SC, e Silva et al. (2010), ambos tendo encontrado 44 espécies.

A curva de acumulação de espécies (Figura 2) indicou tendência para a estabilização, uma vez que, com o acréscimo da última parcela, ocorreu um incremento de $6,7 \%$ na área amostrada, acompanhado pelo aumento de apenas 1,63\% no número de espécies, o que demonstra que a amostragem foi adequada para caracterização florística da área.

As famílias com maior riqueza de espécies em toda a área foram: Myrtaceae (nove espécies), Solanaceae (seis espécies), Aquifoliaceae (quatro espécies), Asteraceae, Lauraceae, Salicaceae e Sapindaceae (três espécies cada) (Tabela 1). A riqueza destas famílias está de acordo com o padrão observado em outros levantamentos em Floresta Ombrófila Mista, que constataram Myrtaceae, Lauraceae, Salicaceae, Sapindaceae, Euphorbiaceae e Asteraceae como famílias de maior riqueza (SEGER et al., 2005). A predominância de espécies

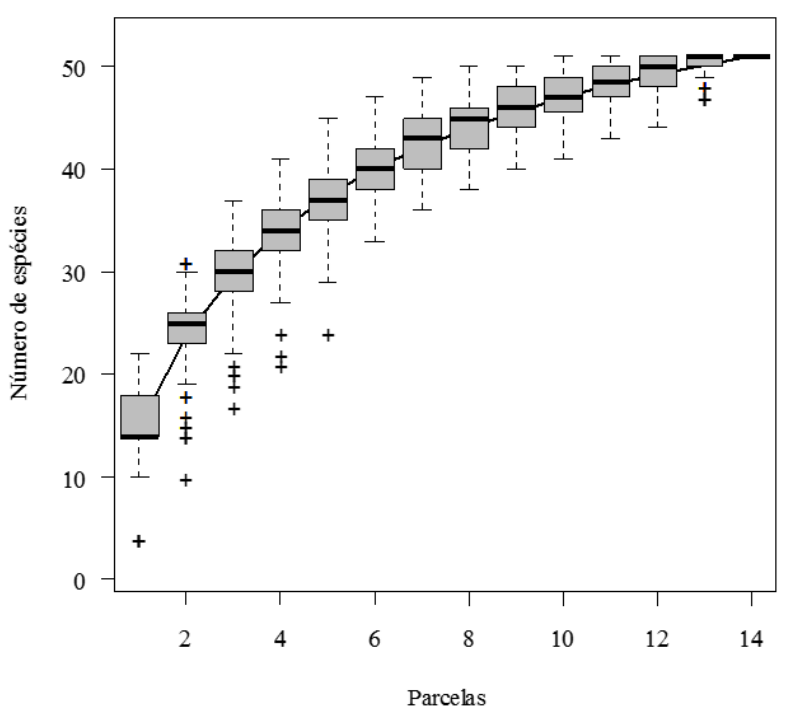

FIGURA 2: Curva de acumulação de espécies do componente regenerativo da comunidade arbórea em um fragmento de Floresta Ombrófila Mista em Lages - SC. A distribuição dos valores de riqueza paras as unidades amostrais é ilustrado por meio de gráficos do tipo "boxplot".

FIGURE 2: Tree species accumulation curve of the tree species regenerative component in an Araucaria Forest fragment in the municipality of Lages, SC state. The distribution of richness values for the sampling plots is showed by boxplot,

da família Myrtaceae também é recorrente em fragmentos de Floresta Ombrófila Mista, como, por exemplo, demonstrado nos trabalhos de Nascimento et al. (2001), Higuchi et al. (2012 e 2013) e Silva et al. (2012).

A maioria das espécies que ocorreu no setor CT (60\%) também foi observada no setor ST (Tabela 1). No entanto, o oposto não se repetiu, uma vez que apenas $38,10 \%$ das espécies do setor ST ocorrem também em CT. Este resultado pode indicar maior seletividade no setor $\mathrm{CT}$, onde ocorre a regeneração somente de espécies tolerantes a este ambiente.

Os indivíduos regenerantes mensurados no setor CT pertencem a 25 espécies, 23 gêneros e 16 famílias botânicas, apresentando uma densidade de $14.133 \mathrm{ind} . /$ ha, que totalizaram uma área basal de $9,03 \mathrm{~m}^{2} /$ ha. Já os indivíduos mensurados no setor ST pertencem a 42 espécies, 31 gêneros de 23 famílias, com uma densidade de 26.350 ind./ ha, totalizando uma área basal de $5,88 \mathrm{~m}^{2} / \mathrm{ha}$. 
TABELA 1: Florística do componente regenerativo (CAP $\leq 15 \mathrm{~cm}$ e altura $\geq 25 \mathrm{~cm}$ ) de uma comunidade arbórea nos setores pós-mortalidade da taquara (CT) e com dossel contínuo do componente adulto (ST) em um fragmento de Floresta Ombrófila Mista em Lages - SC.

TABLE 1: Floristic of tree regenerative component (DBH $\leq 15 \mathrm{~cm}$ e altura $\geq 25 \mathrm{~cm}$ ) in sectors with bamboo die-off (CT) and with continuous canopy cover (ST), in an Araucaria Forest fragment, in the municipality of Lages, SC state.

\begin{tabular}{|c|c|c|c|}
\hline \multirow{2}{*}{ FAMÍLIA } & \multirow{2}{*}{ ESPÉCIE } & \multicolumn{2}{|c|}{ SETOR } \\
\hline & & CT & ST \\
\hline ANNONACEAE & Annona rugulosa (Schltdl.) H.Rainer & $\mathrm{x}$ & $\mathrm{x}$ \\
\hline \multirow{4}{*}{ AQUIFOLIACEAE } & Ilex dumosa Reissek & & $\mathrm{x}$ \\
\hline & Ilex paraguariensis A.St.-Hil. & $\mathrm{x}$ & $\mathrm{x}$ \\
\hline & Ilex taubertiana Loes. & & $\mathrm{x}$ \\
\hline & Ilex theezans Mart. ex Reissek & & $\mathrm{x}$ \\
\hline ARAUCARIACEAE & Araucaria angustifolia (Bertol.) Kuntze & & $\mathrm{x}$ \\
\hline \multirow{3}{*}{ ASTERACEAE } & Dasyphyllum tomentosum (Spreng.) Cabrera & $\mathrm{x}$ & $\mathrm{x}$ \\
\hline & Piptocarpha angustifolia Dusén & $\mathrm{x}$ & \\
\hline & Vernonanthura discolor (Spreng.) H.Rob. & $\mathrm{x}$ & \\
\hline BIGNONIACEAE & Jacaranda puberula Cham. & $\mathrm{x}$ & $\mathrm{x}$ \\
\hline CANELLACEAE & Cinnamodendron dinisii Schwacke & & $\mathrm{x}$ \\
\hline CELASTRACEAE & Maytenus dasyclada Mart. & $\mathrm{x}$ & $\mathrm{x}$ \\
\hline CLETHRACEAE & Clethra scabra Pers. & $\mathrm{x}$ & $\mathrm{x}$ \\
\hline ELAEOCARPACEAE & Sloanea monosperma Vell. & & $\mathrm{x}$ \\
\hline ERYTHROXYLACEAE & Erythroxylum deciduum A.St.-Hil. & & $\mathrm{x}$ \\
\hline EUPHORBIACEAE & Sebastiania commersoniana (Baill.) L.B.Sm. \& Downs & & $\mathrm{x}$ \\
\hline FABACEAE & Mimosa scabrella Benth. & $\mathrm{x}$ & \\
\hline \multirow{3}{*}{ LAURACEAE } & Nectandra lanceolata Nees & & $\mathrm{x}$ \\
\hline & Ocotea puberula (Rich.) Nees & $\mathrm{x}$ & $\mathrm{x}$ \\
\hline & Ocotea pulchella Mart. & & $\mathrm{x}$ \\
\hline LOGANIACEAE & Strychnos brasiliensis (Spreng.) Mart. & $\mathrm{x}$ & $\mathrm{x}$ \\
\hline \multirow{2}{*}{ MELASTOMATACEAE } & Miconia cinerascens Miq. & & $\mathrm{x}$ \\
\hline & Miconia ramboi Brade & & $\mathrm{x}$ \\
\hline MELIACEAE & Cabralea canjerana (Vell.) Mart. & $\mathrm{x}$ & \\
\hline \multirow{9}{*}{ MYRTACEAE } & Blepharocalyx salicifolius (Kunth) O.Berg & $\mathrm{x}$ & $\mathrm{x}$ \\
\hline & Calyptranthes concinna DC. & & $\mathrm{x}$ \\
\hline & Eugenia pluriflora DC. & $\mathrm{x}$ & $\mathrm{x}$ \\
\hline & Myrceugenia ovata (Hook. \& Arn.) O.Berg & & $\mathrm{x}$ \\
\hline & Myrcia hatschbachii D.Legrand & & $\mathrm{x}$ \\
\hline & Myrcia palustris DC. & & $\mathrm{x}$ \\
\hline & Myrcia splendens (Sw.) DC. & $\mathrm{x}$ & $\mathrm{x}$ \\
\hline & Myrcia sp. & & $\mathrm{x}$ \\
\hline & Myrtaceae sp.1 & & $\mathrm{x}$ \\
\hline \multirow{2}{*}{ MYRSINACEAE } & Myrsine coriacea (Sw.) Roem. \& Schult. & & $\mathrm{x}$ \\
\hline & Myrsine lorentziana (Mez) Arechav. & $\mathrm{x}$ & $\mathrm{x}$ \\
\hline
\end{tabular}


TABELA 1: Continuação ...

TABLE 1: Continued ...

\begin{tabular}{|c|c|c|c|}
\hline \multirow{2}{*}{ FAMÍLIA } & \multirow{2}{*}{ ESPÉCIE } & \multicolumn{2}{|c|}{ SETOR } \\
\hline & & $\mathrm{CT}$ & ST \\
\hline PROTEACEAE & Roupala montana Aubl. & $\mathrm{x}$ & $\mathrm{x}$ \\
\hline ROSACEAE & Prunus myrtifolia (L.) Urb. & & $\mathrm{x}$ \\
\hline \multirow{3}{*}{ SALICACEAE } & Banara tomentosa Clos. & & $\mathrm{x}$ \\
\hline & Casearia decandra Jacq. & $\mathrm{x}$ & $\mathrm{x}$ \\
\hline & Casearia obliqua Spreng. & & $\mathrm{x}$ \\
\hline \multirow{3}{*}{ SAPINDACEAE } & Allophylus guaraniticus (A.St.-Hil.) Radlk. & & $\mathrm{x}$ \\
\hline & Cupania vernalis Cambess. & $\mathrm{x}$ & $\mathrm{x}$ \\
\hline & Matayba elaeagnoides Radlk. & $\mathrm{x}$ & $\mathrm{x}$ \\
\hline \multirow{6}{*}{ SOLANACEAE } & Solanum mauritianum Scop. & & \\
\hline & Solanum pabstii L.B.Sm. \& Downs & & $\mathrm{x}$ \\
\hline & Solanum paranense Dusén & $\mathrm{x}$ & \\
\hline & Solanum pseudoquina A.St.-Hil. & $\mathrm{x}$ & \\
\hline & Solanum sp. & $\mathrm{x}$ & \\
\hline & Solanum variabile Mart. & $\mathrm{x}$ & \\
\hline SYMPLOCACEAE & Symplocos uniflora (Pohl) Benth. & & $\mathrm{x}$ \\
\hline WINTERACEAE & Drimys brasiliensis Miers. & & $\mathrm{x}$ \\
\hline Total de espécies & & 25 & 42 \\
\hline
\end{tabular}

$\mathrm{O}$ setor ST apresentou um Índice de diversidade de Shannon (H') e Equabilidade de Pielou (J), respectivamente, de 2,73 e 0,76 , enquanto o setor CT obteve H' de 2,31 e J de 0,73 (Tabela 2). Quando a riqueza entre os locais é comparada por meio da técnica de rarefação, observa-se que o número de espécies do setor ST $(33,04 \pm 1,62)$ foi superior ao do setor CT (25). Ressalta-se que o valor encontrado no setor CT não apresenta desvio padrão, pois se trata do valor absoluto de espécies encontrado. Este resultado difere do encontrado por Budke et al. (2010), no qual foi observada maior riqueza de espécies arbóreas nos locais abertos dois anos após floração sincronizada e posterior mortalidade da taquara.

As cinco espécies de maior valor de importância no setor CT foram: Solanum variabile (29,01\%), Piptocarpha angustifolia (18,29\%), Mimosa scabrella (8,63\%), Jacaranda puberula $(4,24 \%)$ e Solanum pseudoquina $(3,90 \%)$ (Tabela $3)$, todas podendo ser classificadas como pioneiras e clímax exigentes de luz. No setor ST, as espécies Myrsine lorentziana (14,30\%), Casearia decandra 0,7052 e 0,1948 , que explicam $78,69 \%$ da variação dos dados encontrados. O eixo 1 sintetizou mais da
TABELA 2: Descritores florísticos e estruturais avaliados para o componente regenerativo da comunidade arbórea em um fragmento de Floresta Ombrófila Mista Montana em Lages - SC, avaliados no setor com taquara (CT) e sem taquara (ST).

TABLE 2: Floristic and structural descriptors evalated for the tree community regenerative component in a Montane Araucaria forest fragment, in the municipality of Lages, SC state, considering sectors with bamboo dieoff (CT) and continuous canopy cover (ST).

\begin{tabular}{lcc}
\hline \multicolumn{1}{c}{ Descritores avaliados } & CT & ST \\
\hline Número de espécies & 25 & 42 \\
Número de gêneros & 23 & 31 \\
Número de famílias & 16 & 23 \\
Índice de diversidade de Shannon (H') & 2,31 & 2,73 \\
Equabilidade de Pielou (J) & 0,73 & 0,76 \\
Número de indivíduos/há & 14.133 & 26.350 \\
Área basal/ha & 9,03 & 5,88 \\
\hline
\end{tabular}


TABELA 3: Descritores fitossociológicos do componente regenerativo da comunidade arbórea, ordenados de forma decrescente pelo valor de importância (VI), do setor com taquaras, em um fragmento de Floresta Ombrófila Mista Montana em Lages - SC.

TABLE 3: Phytosociological descriptors of tree community regenerative component, in die-off bamboo sector, ranked in descending order of importance value (VI), in a montane Araucaria Forest fragment, in the municipality of Lages, SC state.

\begin{tabular}{|c|c|c|c|c|c|c|c|c|}
\hline Espécies & GE & DA & DR & DoA & DoR & FA & FR & VI \\
\hline Solanum variabile & $\mathrm{P}$ & 4933 & 34,91 & 4,0272 & 43,80 & 100,0 & 8,33 & 29,01 \\
\hline Piptocarpha angustifolia & $\mathrm{P}$ & 3000 & 21,23 & 2,3302 & 25,31 & 100,0 & 8,33 & 18,29 \\
\hline Mimosa scabrella & $\mathrm{P}$ & 600 & 4,25 & 1,0548 & 13,30 & 100,0 & 8,33 & 8,63 \\
\hline Jacaranda puberula & $\mathrm{CL}$ & 533 & 3,77 & 0,1853 & 2,01 & 83,0 & 6,94 & 4,24 \\
\hline Solanum pseudoquina & $\mathrm{CL}$ & 600 & 4,25 & 0,3024 & 3,29 & 50,0 & 4,17 & 3,90 \\
\hline Ocotea puberula & $\mathrm{CS}$ & 533 & 3,77 & 0,0585 & 0,64 & 67,0 & 5,56 & 3,32 \\
\hline Cupania vernalis & $\mathrm{CS}$ & 400 & 2,83 & 0,0455 & 0,49 & 67,0 & 5,56 & 2,96 \\
\hline Solanum paranense & $\mathrm{P}$ & 200 & 1,42 & 0,2785 & 3,03 & 50,0 & 4,17 & 2,87 \\
\hline Vernonanthura discolor & $\mathrm{P}$ & 333 & 2,36 & 0,1555 & 1,69 & 50,0 & 4,17 & 2,74 \\
\hline Roupala montana & $\mathrm{CS}$ & 467 & 3,30 & 0,0534 & 0,58 & 50,0 & 4,17 & 2,68 \\
\hline Matayba elaeagnoides & $\mathrm{CS}$ & 267 & 1,89 & 0,0153 & 0,17 & 67,0 & 5,56 & 2,54 \\
\hline Annona rugulosa & $\mathrm{CS}$ & 200 & 1,42 & 0,1755 & 1,91 & 50,0 & 4,17 & 2,50 \\
\hline Casearia decandra & $\mathrm{CS}$ & 267 & 1,89 & 0,0958 & 1,04 & 50,0 & 4,17 & 2,37 \\
\hline Myrcia splendens & $\mathrm{CS}$ & 267 & 1,89 & 0,0275 & 0,30 & 50,0 & 4,17 & 2,12 \\
\hline Não identificadas & - & 200 & 1,42 & 0,0606 & 0,96 & 33,0 & 2,78 & 1,72 \\
\hline Maytenus dasyclada & $\mathrm{CS}$ & 133 & 0,94 & 0,0346 & 0,38 & 33,0 & 2,78 & 1,37 \\
\hline Cabralea canjerana & $\mathrm{CS}$ & 133 & 0,94 & 0,0204 & 0,22 & 33,0 & 2,78 & 1,31 \\
\hline Clethra scabra & $\mathrm{CL}$ & 333 & 2,36 & 0,0157 & 0,17 & 17,0 & 1,39 & 1,31 \\
\hline Myrsine lorentziana & $\mathrm{CL}$ & 133 & 0,94 & 0,0051 & 0,06 & 33,0 & 2,78 & 1,26 \\
\hline Solanum mauritianum & $\mathrm{P}$ & 133 & 0,94 & 0,0252 & 0,27 & 17,0 & 1,39 & 0,87 \\
\hline Ilex paraguariensis & $\mathrm{CS}$ & 133 & 0,94 & 0,0039 & 0,04 & 17,0 & 1,39 & 0,79 \\
\hline Solanum sp. & - & 67 & 0,47 & 0,0327 & 0,36 & 17 & 1,39 & 0,74 \\
\hline Strychnos brasiliensis & $\mathrm{CS}$ & 67 & 0,47 & 0,0118 & 0,13 & 17,0 & 1,39 & 0,66 \\
\hline Dasyphyllum tomentosum & $\mathrm{CL}$ & 67 & 0,47 & 0,0075 & 0,08 & 17,0 & 1,39 & 0,65 \\
\hline Blepharocalyx salicifolius & $\mathrm{CS}$ & 67 & 0,47 & 0,0042 & 0,05 & 17,0 & 1,39 & 0,64 \\
\hline Eugenia pluriflora & $\mathrm{CS}$ & 67 & 0,47 & 0,0026 & 0,03 & 17,0 & 1,39 & 0,63 \\
\hline
\end{tabular}

Em que: $\mathrm{GE}$ = Grupo ecológico (P: pioneiras, CL: clímax exigentes de luz e CS: clímax tolerantes à sombra); $\mathrm{DA}=$ densidade absoluta (ind ha- $\left.{ }^{-1}\right) ; \mathrm{DR}=$ densidade relativa (\%); DoA = dominância absoluta $\left(\mathrm{m}^{2} \mathrm{ha}^{-1}\right)$; DoR $=$ dominância relativa (\%); FA = frequência absoluta (\%); FR = frequência relativa (\%); VI = valor de importância (\%).

(12,72\%), Cinnamodendron dinisii (10,68\%), Drimys brasiliensis $(8,74 \%)$ e Ilex paraguariensis $(5,49 \%)$ foram as que tiveram maior valor de VI (Tabela 4), todas como clímax tolerantes ao sombreamento, frequentemente encontradas no sub-bosque de florestas. O presente resultado mostra que, apesar de estarem pouco representadas durante a fase com predominância de bambu, como demonstrado por alguns estudos (e.g. TABARELLI e MANTOVANI, 2000), as espécies pioneiras podem dominar a regeneração após cinco anos do evento de mortalidade deste. A dominância de espécies iniciais após a mortalidade do bambu pode ser explicada pela maior disponibilidade de luminosidade que estas espécies estariam expostas (BUDKE et al., 2010; LARPKERN et al., 2011), favorecendo o seu desenvolvimento.

A análise de correspondência retificada (DCA) (Figura 3) apresentou autovalores para o primeiro e segundo eixos, respectivamente, de 
TABELA 4: Descritores fitossociológicos do componente regenerativo da comunidade arbórea, ordenados de forma decrescente pelo valor de importância (VI), do setor sem taquaras, em um fragmento de Floresta Ombrófila Mista Montana em Lages - SC.

TABLE 4: Phytosociological descriptors of tree community regenerative component, in continuous canopy cover sector, ranked in descending order of importance value (VI), in a montane Araucaria Forest fragment, in the municipality of Lages, SC state.

\begin{tabular}{|c|c|c|c|c|c|c|c|c|}
\hline Espécies & GE & $\mathrm{DA}$ & DR & DoA & DoR & $\mathrm{FA}$ & FR & VI \\
\hline Myrsine lorentziana & $\mathrm{CS}$ & 6550 & 24,86 & 0,7279 & 12,38 & 100,0 & 5,67 & 14,30 \\
\hline Casearia decandra & $\mathrm{CS}$ & 3400 & 12,90 & 1,1513 & 19,58 & 100,0 & 5,67 & 12,72 \\
\hline Cinnamodendron dinisii & $\mathrm{CS}$ & 500 & 1,90 & 1,5646 & 26,61 & 63,0 & 3,55 & 10,68 \\
\hline Drimys brasiliensis & $\mathrm{CS}$ & 2050 & 7,78 & 0,7500 & 12,76 & 100,0 & 5,67 & 8,74 \\
\hline Ilex paraguariensis & $\mathrm{CS}$ & 1000 & 3,80 & 0,4530 & 7,70 & 88,0 & 4,96 & 5,49 \\
\hline Matayba elaeagnoides & $\mathrm{CS}$ & 2150 & 8,16 & 0,1126 & 1,92 & 100,0 & 5,67 & 5,25 \\
\hline Ilex theezans & $\mathrm{CS}$ & 900 & 3,42 & 0,3126 & 5,32 & 63,0 & 3,55 & 4,09 \\
\hline Ocotea pulchella & $\mathrm{CS}$ & 1300 & 4,93 & 0,1470 & 2,50 & 75,0 & 4,26 & 3,90 \\
\hline Jacaranda puberula & $\mathrm{CL}$ & 700 & 2,66 & 0,0730 & 1,24 & 75,0 & 4,26 & 2,72 \\
\hline Symplocos uniflora & $\mathrm{CL}$ & 1250 & 4,74 & 0,0145 & 0,25 & 38,0 & 2,13 & 2,37 \\
\hline Myrcia palustris & $\mathrm{CS}$ & 650 & 2,47 & 0,0269 & 0,46 & 63,0 & 3,55 & 2,16 \\
\hline Roupala montana & $\mathrm{CS}$ & 400 & 1,52 & 0,0355 & 0,60 & 75,0 & 4,26 & 2,13 \\
\hline Cupania vernalis & $\mathrm{CS}$ & 700 & 2,66 & 0,0400 & 0,68 & 50,0 & 2,84 & 2,06 \\
\hline Araucaria angustifolia & $\mathrm{CL}$ & 450 & 1,71 & 0,0283 & 0,48 & 63,0 & 3,55 & 1,91 \\
\hline Annona rugulosa & $\mathrm{CS}$ & 600 & 2,28 & 0,0889 & 1,51 & 25,0 & 1,42 & 1,74 \\
\hline Myrcia splendens & $\mathrm{CS}$ & 200 & 0,76 & 0,0791 & 1,35 & 38,0 & 2,13 & 1,41 \\
\hline Myrtaceae sp.1 & - & 250 & 0,95 & 0,0145 & 0,25 & 50,0 & 2,84 & 1,34 \\
\hline Strychnos brasiliensis & $\mathrm{CS}$ & 200 & 0,76 & 0,0129 & 0,22 & 50,0 & 2,84 & 1,27 \\
\hline Calyptranthes concinna & $\mathrm{CS}$ & 300 & 1,14 & 0,0268 & 0,46 & 38,0 & 2,13 & 1,24 \\
\hline Ocotea puberula & $\mathrm{CS}$ & 350 & 1,33 & 0,0115 & 0,20 & 38,0 & 2,13 & 1,23 \\
\hline Miconia cinerascens & $\mathrm{CL}$ & 250 & 0,95 & 0,0130 & 0,22 & 38,0 & 2,13 & 1,10 \\
\hline Banara tomentosa & $\mathrm{CS}$ & 250 & 0,95 & 0,0074 & 0,13 & 38,0 & 2,13 & 1,07 \\
\hline Dasyphyllum tomentosum & $\mathrm{CL}$ & 200 & 0,76 & 0,0059 & 0,10 & 38,0 & 2,13 & 1,00 \\
\hline Não identificadas & - & 200 & 0,76 & 0,0035 & 0,06 & 38,0 & 2,13 & 0,98 \\
\hline Ilex dumosa & $\mathrm{CS}$ & 100 & 0,38 & 0,0387 & 0,66 & 25,0 & 1,42 & 0,82 \\
\hline Eugenia pluriflora & $\mathrm{CS}$ & 150 & 0,57 & 0,0095 & 0,16 & 25,0 & 1,42 & 0,72 \\
\hline Myrsine coriacea & $\mathrm{CL}$ & 150 & 0,57 & 0,0080 & 0,14 & 25,0 & 1,42 & 0,71 \\
\hline Maytenus dasyclada & $\mathrm{CS}$ & 100 & 0,38 & 0,0079 & 0,13 & 25,0 & 1,42 & 0,64 \\
\hline Myrcia sp. & - & 100 & 0,38 & 0,0079 & 0,13 & 25,0 & 1,42 & 0,64 \\
\hline Prunus myrtifolia & $\mathrm{CS}$ & 100 & 0,38 & 0,0024 & 0,04 & 25,0 & 1,42 & 0,61 \\
\hline Sloanea monosperma & $\mathrm{CS}$ & 100 & 0,38 & 0,0020 & 0,03 & 25,0 & 1,42 & 0,61 \\
\hline Ilex taubertiana & $\mathrm{CS}$ & 50 & 0,19 & 0,0428 & 0,73 & 13,0 & 0,71 & 0,54 \\
\hline Myrcia hatschbachii & $\mathrm{CS}$ & 100 & 0,38 & 0,0198 & 0,34 & 13,0 & 0,71 & 0,48 \\
\hline Clethra scabra & $\mathrm{CL}$ & 100 & 0,38 & 0,0051 & 0,09 & 13,0 & 0,71 & 0,39 \\
\hline Sebastiania commersoniana & $\mathrm{CS}$ & 100 & 0,38 & 0,0036 & 0,06 & 13,0 & 0,71 & 0,38 \\
\hline Miconia ramboi & $\mathrm{CL}$ & 50 & 0,19 & 0,0088 & 0,15 & 13,0 & 0,71 & 0,35 \\
\hline
\end{tabular}


TABELA 4: Continuação...

TABLE 4: Continued...

\begin{tabular}{llccccccc}
\hline \multicolumn{1}{c}{ Espécies } & GE & DA & DR & DoA & DoR & FA & FR & VI \\
\hline Nectandra lanceolata & CS & 50 & 0,19 & 0,0066 & 0,11 & 13,0 & 0,71 & 0,34 \\
Allophylus guaraniticus & CS & 50 & 0,19 & 0,0039 & 0,07 & 13,0 & 0,71 & 0,32 \\
Casearia obliqua & CS & 50 & 0,19 & 0,0039 & 0,07 & 13,0 & 0,71 & 0,32 \\
Solanum pabstii & CL & 50 & 0,19 & 0,0032 & 0,05 & 13,0 & 0,71 & 0,32 \\
Blepharocalyx salicifolius & CS & 50 & 0,19 & 0,0025 & 0,04 & 13,0 & 0,71 & 0,31 \\
Myrceugenia ovata & CL & 50 & 0,19 & 0,0019 & 0,03 & 13,0 & 0,71 & 0,31 \\
Erythroxylum deciduum & $\mathrm{CL}$ & 50 & 0,19 & 0,0006 & 0,01 & 13,0 & 0,71 & 0,30 \\
\hline
\end{tabular}

Em que: GE = Grupo ecológico (P: pioneiras, CL: clímax exigentes de luz e CS: clímax tolerantes à sombra); $\mathrm{DA}=$ densidade absoluta (ind ha-1); $\mathrm{DR}=$ densidade relativa (\%); DoA = dominância absoluta $\left(\mathrm{m}^{2} \mathrm{ha}^{-1}\right)$; $\mathrm{DoR}=$ dominância relativa $(\%) ; \mathrm{FA}=$ frequência absoluta $(\%) ; \mathrm{FR}=$ frequência relativa $(\%) ; \mathrm{VI}=$ valor de importância (\%).

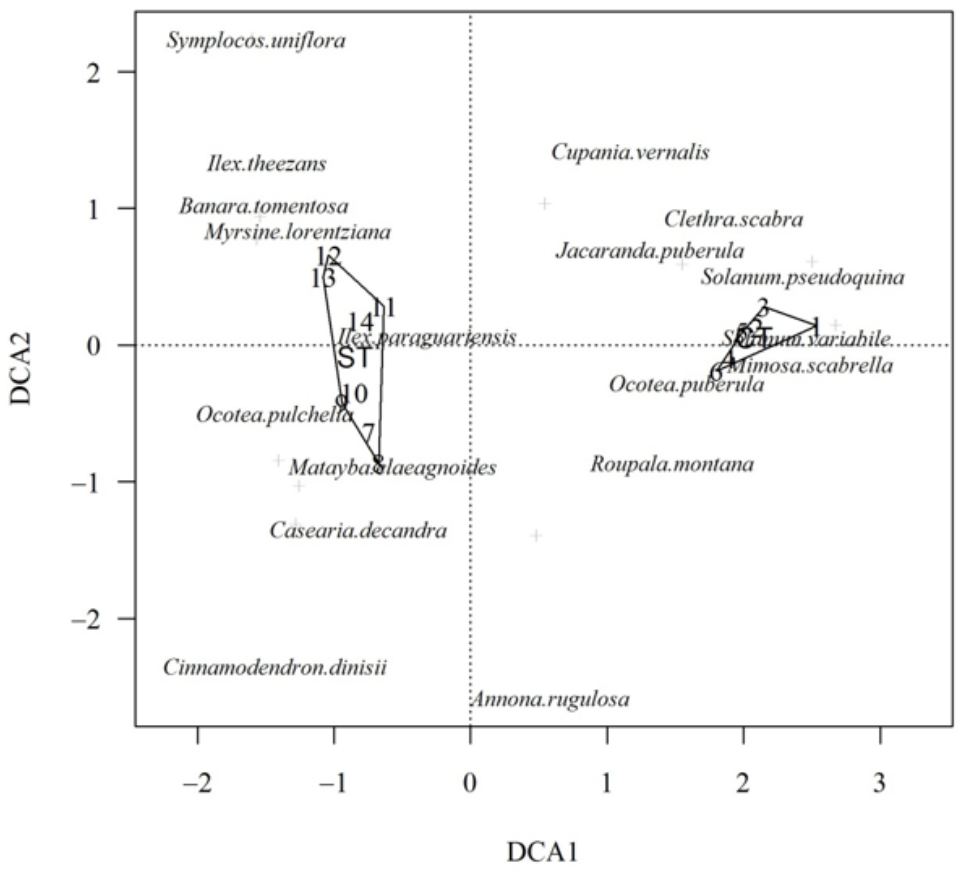

FIGURA 3: Ordenação das parcelas e espécies, por meio da Análise de Correspondência Retificada, em um fragmento de Floresta Ombrófila Mista em Lages - SC. ST = setor sem taquara; CT = setor com taquara; + espécies, de baixa densidade, cujos nomes foram omitidos para facilitar a visualização e interpretação da ordenação.

FIGURE 3: Plots and species ordination, by Detrended Correspondence Analysis, in a montane Araucaria Forest fragment, in the municipality of Lages, SC state. ST = Sector with continuous canopy cover; CT = Sector with bamboo die-off; + Low density omitted species, in order to improve visualization and interpretation of ordination.

metade da variação encontrada $(61,66 \%)$ e separou os dois setores estudados. O elevado autovalor encontrado para este eixo indica elevada substituição de espécie ao longo do gradiente ambiental (TER BRAAK, 1995), de forma que ocorrem várias espécies exclusivas em ambos os setores. $\mathrm{O}$ agrupamento de parcelas formado no lado esquerdo do gráfico da DCA correspondeu ao setor sem taquaras (parcelas 7 a 14) e o agrupamento formado do lado direito, ao setor com taquaras (Parcelas 1 a 6). As espécies mais correlacionadas com o setor ST foram (valores entre parênteses representam 
os "scores" das espécies no eixo de ordenação, produzido pela DCA): Symplocos uniflora $(-1,57)$, Banara tomementosa $(-1,51)$, Ilex theezans $(-1,49)$, Ocotea pulchella $(-1,43)$ e Cinnamodendron dinisii $(-1,43)$. As espécies mais correlacionadas com o agrupamento das parcelas no setor CT foram: Mimosa scabrella $(2,49)$, Solanum variabile $(2,46)$, Solanum pseudoquina (2,43), Clethra scabra $(1,93)$ e Ocotea puberula $(1,58)$. Estas correlações confirmam a predominância de espécies iniciais de sucessão no setor onde ocorreu a mortalidade do maciço de taquara e espécies tardias no setor sem a presença de taquara.

\section{CONCLUSÕES}

Foipossívelobservara dominânciaecológica de espécies pertencentes ao grupo das pioneiras, que ocuparam o espaço liberado pelo maciço de taquara, aproximadamente cinco anos após a mortalidade deste. Em contrapartida, a regeneração natural da área adjacente com dossel contínuo apresentou dominância de espécies arbóreas tolerantes ao sombreamento, típicas do sub-bosque. Dessa forma, os resultados demonstraram que, em pequena escala espacial, eventos de mortalidade sincronizada de maciços de taquara representam uma importante fonte de heterogeneidade florística e estrutural da regeneração natural de espécies arbóreas em florestas. Com isso, é possível estabelecer uma analogia entre clareiras naturais formadas pela queda de árvores e o espaço liberado pela morte de maciços de taquara.

\section{REFERÊNCIAS BIBLIOGRÁFICAS}

BROKAW, N. V. L. Gap-phase regeneration of three pioneer tree species in a tropical forest. Journal of Ecology, Oxford, v. 75, p. 9-19. 1987.

BUDKE, J. C. et al. Bamboo dieback and tree regeneration responses in a subtropical forest in South America. Forest Ecology and Management, Amsterdam, v. 260, p. 1345-1349. 2010.

CALDATO, S. L. et al. Estudo da regeneração natural, banco de sementes e chuva de sementes na reserva genética florestal de Caçador, SC. Ciência Florestal, Santa Maria, v. 6, n. 1, p. 27-38. 1996.

GUILHERME, F. A. G. et al. Effects of flooding regime and woody bamboos on tree community dynamics in a section of tropical semideciduous forest in South-Eastern Brazil. Plant Ecology,
Dordrecht, v. 174, p. 19-36. 2004.

HIGUCHI, P. et al. Spatial patterns of a tropical tree species growing under an eucalyptus plantation in South-East Brazil. Brazilian Journal of Biology, São Carlos, v. 70, n. 2, p. 271-277. 2010.

HIGUCHI, P. et al. Influência de variáveis ambientais sobre o padrão estrutural e florístico do componente arbóreo em um fragmento de Floresta Ombrófila Mista Montana, em Lages, SC. Ciência Florestal, Santa Maria, v. 22, n. 1, p. 79-90, 2012. HIGUCHI, P. et al. Florística e estrutura do componente arbóreo e análise ambiental de um fragmento de Floresta Ombrófila Mista Altomontana no município de Painel, SC. Ciência Florestal, Santa Maria, v. 23, n. 1, p. 153-164, 2013.

IBGE. Manual técnico da vegetação brasileira. Rio de Janeiro: Fundação Instituto Brasileiro de Geografia e Estatística, 1992. 92 p. (. Série: Manuais técnicos em geociências n.1).

LARPKERN, P.; MOE, S. R.; TOTLAND, O. Bamboo dominance reduces tree regeneration in a disturbed tropical forest. Oecologia, Berlin, v. 165, n. 1, p. 165-168. 2011.

LIEBSCH, D.; REGINATO, M. Florescimento e frutificação de Merostachys skvortzovii Sendulsky (taquara-lixa) no estado do Paraná. Iheringia, Porto Alegre, v. 64, n. 1, p. 53-56. 2009.

MAROD, D. et al. Structural dynamics of a natural mixed deciduous forest in western Thailand. Journal of Vegetation Science, v. 10, n. 6, p. 777786. 1999.

MONTGOMERY, R. A.; CHAZDON, R. L. Light gradient partitioning by tropical tree seedlings in the absence of canopy gaps. Oecologia, Berlin, v. 131, p. 165-174. 2002.

MULLER-DOMBOIS, D.; ELLENBERG, H. Aims and of vegetation ecology. New York: John Wiley \& Sons, 1974. $547 \mathrm{p}$.

NASCIMENTO, A. R. T.; LONGHI, S. J.; BRENA, A. D. Estrutura e padrões de distribuição espacial de espécies arbóreas em uma amostra de Floresta Ombrófila Mista em Nova Prata, RS. Ciência Florestal, Santa Maria, v. 11, n. 1, p. 105-119. 2001. OKSANEN, J. et al. Vegan: Community Ecology Package. R package version 1.17-2. Disponível em: $<$ (http://CRAN.R-project.org/package=vegan $)>$ Acesso em: 15 de dezembro de 2010.

R DEVELOPMENT CORE TEAM. R: A language and environment for statistical computing. R Foundation for Statistical Computing, 2010. Disponível em: <(http://www.R-project.org)> 
Acesso em: 15 de dezembro de 2010.

ROSSI, J. P. Rich: species richness estimation and comparison. R package version 0.1. Disponível em: $<$ (http://CRAN.R-project.org/package=rich) $>$ Acesso em 15 de abril de 2011.

SCHMIDT, R. A tribo Bambuseae Nees (Poaceae, Bambusoideae) no Rio Grande do Sul, Brasil. 2008. 137 f. Dissertação (Mestrado em Botânica) Universidade Federal do Rio Grande do Sul, Porto Alegre.

SCHNITZER, S.; DALLING, J. W.; CARSON, W. P. The impact of lianas on tree regeneration in tropical forest canopy gaps: evidence for an alternative pathway of gap-phase regeneration. Journal of Ecology, Oxford, v. 88, n. 4, p. 655-666. 2000.

SEGER, C. D. et al. Levantamento florístico e análise fitossociológica de um remanescente de floresta ombrófila mista localizado no município de Pinhais, Paraná-Brasil. Floresta, Curitiba, v. 35, n. 2, p. 291-302. 2005.

SILVA, M. M.; GANADE, G. M. S.; BACKES, A. Regeneração natural em um remanescentes de Floresta Ombrófila Mista, na Floresta Nacional de São Francisco de Paula, Rio Grande do Sul,
Brasil. Pesquisas Botânicas, São Leopoldo, v. 61, p. 259-278, 2010.

SILVA, A. C. et al. Relações florísticas e fitossociologia de uma Floresta Ombrófila Mista Montana secundária em Lages, Santa Catarina. Ciência Florestal, Santa Maria, v.22, n.1, p. 193-206, 2012.

SWAINE, M.D.; WHITMORE, T.C. On the definition of ecological species groups in tropical rain forests. Vegetatio, Dordrecht, v. 75, n.1, p. 81-86, 1988.

TABARELLI, M.; MANTOVANI, W. Gapphase regeneration in a tropical montane forest: the effects of gap structure and bamboo species.

Plant Ecology, Dordrecht, v. 148, p. 149-155. 2000.

TER BRAAK, C. J. F. Ordination. In: JONGMAN, R. H. G.; TER BRAAK, C. J. F.; VAN TONGEREN, O.F. R. Data analysis in community and landscape ecology. Cambridge: Cambridge University Press, 1995. p. 91-173.

VEENENDAAL, E. M. et al. Response of west African forest tree seedlings to irradiance and soil fertility. Functional Ecology, London, v. 10, n. 4. p. 501-511. 1996. 